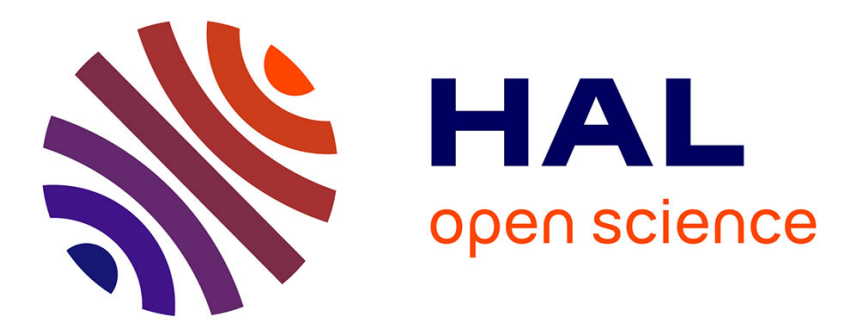

\title{
Eye eccentricity modifies the perception of whole-body rotation
}

\author{
Gaëlle Quarck, Léna Lhuisset, Olivier M Etard, Pierre F Denise
}

\section{To cite this version:}

Gaëlle Quarck, Léna Lhuisset, Olivier M Etard, Pierre F Denise. Eye eccentricity modifies the perception of whole-body rotation. Experimental Brain Research, 2009, 196 (2), pp.295-301. 10.1007/s00221-009-1828-z . hal-02364427

\section{HAL Id: hal-02364427 \\ https://hal.science/hal-02364427}

Submitted on 19 Nov 2019

HAL is a multi-disciplinary open access archive for the deposit and dissemination of scientific research documents, whether they are published or not. The documents may come from teaching and research institutions in France or abroad, or from public or private research centers.
L'archive ouverte pluridisciplinaire HAL, est destinée au dépôt et à la diffusion de documents scientifiques de niveau recherche, publiés ou non, émanant des établissements d'enseignement et de recherche français ou étrangers, des laboratoires publics ou privés. 


\title{
Eye eccentricity modifies the perception of whole-body rotation
}

\author{
G. Quarck*, L. Lhuisset, O. Etard, P. Denise
}

Univ Caen, UPRES-EA 3917 - Mobilité: cognition et temporalité, Faculté de Médecine, Caen Cedex, F-14 032, France

* Corresponding author

Tel: +33231068214

Fax: +33231068219

e-mail address: gaelle.quarck@unicaen.fr 


\section{Abstract}

In order to explore the effect of gaze orientation on whole body rotation perception, 10 healthy participants were rotated in the dark while fixating on a visual target located either straight ahead or $15^{\circ}$ to the right. A vestibular-memory contingent saccade paradigm was used to estimate the rotation perception. The results attest to the participants' ability to accurately perceive their rotation, based solely on the intrinsic inputs (somesthetic and mainly vestibular), since the correlation between the imposed body rotation and the saccade amplitude was significant and positive. However, the rotation perception was less accurate and of lesser magnitude when the gaze was deviated in the opposite direction to the rotation than when it was either straight ahead or deviated in the direction of the rotation. This can be interpreted as the perceptual equivalent of Alexander's law.

\section{Keywords:}

Vestibule, Vestibulo-ocular reflex, Orientation, Saccades, Eye movements, Alexander's law 


\section{Introduction}

Self-orientation and localisation in space is ensured through multimodal integration of sensory and motor signals such as visual, somesthetic and vestibular information as well as efference copies, depending on the context. When body motion occurs in darkness, the vestibular apparatus is of primary importance since it detects angular and linear acceleration of the head through semicircular canals and otoliths respectively; the head displacement is then retrieved by the brain through time integration of these signals. Several experimental procedures have been used to estimate the implication of the vestibular system in passive whole-body movement perception. In these studies, participants were passively rotated in darkness and were then asked to estimate the amplitude of their displacement. The estimation was assessed either verbally (Brown 1966; Blouin et al. 1995b; Jurgens et al. 1999) or through the reproduction of the perceived movement with either self-control re-orienting movement in response to rotation (Metcalfe and Gresty 1992; Siegler et al. 2000; Israel et al. 2006), manual pointing or remote control tasks (Blouin et al. 1995a; Mergner et al. 2001) or the so-called vestibular-memory contingent saccade task (Bloomberg et al. 1988; Bloomberg et al. 1991; Israel et al. 1993; Blouin et al. 1997; Mergner et al. 1998). Results showed that passive body rotation in darkness seems to be well reproduced through ocular saccades. Different studies (Bloomberg et al. 1988; Bloomberg et al. 1991; Israel et al. 1993; Blouin et al. 1997) showed a high correlation between the amplitude of the vestibular-memory contingent saccade and the amplitude of the rotation. In these studies, participants first fixated a centrally placed earth-fixed target and were then submitted to whole-body rotation in darkness while maintaining ocular fixation on a head-fixed target (to suppress the Vestibulo-ocular reflex). Upon completion of rotation participants then had to execute an ocular saccade toward the estimated initial location of the earth-fixed target. It is known, however, that the central treatment of the vestibular input can be affected by the position of the eyes in the orbit. In fact, a phenomenon known as the Alexander's law (see 
(Robinson et al. 1984; Evanoff and Lackner 1986)) states that the nystagmus is more intense when the gaze is oriented in the quick-phase direction (i.e. same direction as the rotation) than in the slow-phase direction (i.e. opposite direction to the rotation). Since the eye position relative to rotation direction affects central vestibular processing, one can reasonably question the effect of this parameter on the perception derived from peripheral vestibular input. Yet, in studies using the vestibular-memory contingent saccade procedure (Bloomberg et al. 1988; Bloomberg et al. 1991; Blouin et al. 1997; Mergner et al. 1998), the gaze was always directed straight ahead during the rotation and therefore the effect of eye position relative to rotation direction on the perception of this rotation could not be investigated. The aim of our study was to address this question, by applying whole-body rotations to normal participants and studying the effect of initial eye position (either straight ahead or $15^{\circ}$ to the right) on vestibular memory contingent saccade performance. .

\section{Material and method}

\section{Participants}

Ten young adults (mean age $21.1 \pm 1.4$ years; 3 women and 7 men) took part in this study. They had normal or corrected to normal vision and didn't present any vestibular pathology, as assessed through an otological physical examination. Local ethics committee approval and a written informed consent from the participants were obtained. The study was conducted according to guidelines set by the Helsinki Declaration for the treatment of experimental participants.

\section{Apparatus and material}

Participants sat in the darkness on a computer controlled motorized rotatory chair with their head upright and restrained with a bite-bar so that the chair rotation would induce an associated head and body rotation. Two light emitting diodes (LEDs) were fixed onto the chair (chair-fixed targets) at a distance of 1 metre in front of the participants: one was positioned straight ahead and the other $15^{\circ}$ 
to the right. Given that the LEDs were fixed onto the rotating chair, they were stationary relative to the participants.

Horizontal eye movements (bitemporal derivation) and vertical eye movements of one eye were measured by AC electro-oculography (EOG). All testing was performed with the participant's eyes open in darkness. A full calibration sequence (steps of $5^{\circ}$ from $0^{\circ}$ to $25^{\circ}$ in each four directions) was applied at the start, in the middle and at the end of each experimental session. Furthermore, a horizontal eye movement calibration sequence $\left(+15^{\circ} /-15^{\circ}\right)$ was applied systematically after each trial. This second calibration sequence was carried out to make sure that the former one remained valid throughout the experimental session. Since this was indeed the case, the full calibration sequence was used to calibrate the data. Chair position, horizontal and vertical EOG, and LED signals were sampled on-line at $100 \mathrm{~Hz}$ (Gould amplificator, $0.05 \mathrm{~Hz}$ highpass cutoff frequency) and recorded on the same PC that controlled chair rotation (software Asamo developed in our lab).

\section{Task and procedure}

Participants were subjected to rotations randomly ranging from 3 to 30 degrees to the left or to the right, meaning that the same stimulation was never presented twice. After an acceleration of $10^{\circ} \mathrm{s}^{-2}$ for up to 1 second, subjects were rotated at a constant velocity of $10^{\circ} \mathrm{s}^{-1}$ for a maximum of 2 seconds, then decelerated at $-10^{\circ} \mathrm{s}^{-2}$. For amplitude less than $10^{\circ}$, there was no constant velocity phase and acceleration and deceleration phases were shorter. Participants performed 20 to 23 trials in each fixation condition (chair-fixed target straight ahead or $15^{\circ}$ to the right). One second before the beginning of the rotation, one of the 2 chair-fixed targets was illuminated. Participants were instructed to fix their gaze on the chair-fixed target until it was extinguished at the end of the rotation. The participant's task was then to direct their gaze towards the estimated initial spatial location of the fixation point as accurately as possible. The participants were allowed to make several successive saccades to improve their accuracy, however, they mostly performed only one 
saccade. After a delay of $1500 \mathrm{~ms}$, the LED, in its actual position after the rotation, was lit again and the participants had to foveate it. This foveation was always performed in one saccade of equal amplitude of the cumulative saccade size but in the opposite direction. We chose to analyse the amplitude of this saccade due to the fact that it was more precise and simpler to quantify.

\section{Data analysis}

The data were submitted to different analyses (linear regression, correlation and ANOVAs) depending on the question of interest. The details of these analyses are provided in the Results section whereappropriate. For the ANOVAs, Greenhouse-Geisser correction was applied when the Epsilon value was smaller than 1 (Greenhouse and Geisser 1959); see also (Winer 1971). Because the correction did not modify the outcome of these analyses, we reported the data using the original degrees of freedom. All significant main effects and interactions were further delineated using the Newman-Keuls technique. Statistical tests were performed using Statistica Software® (Statsoft, Tulsa, USA). Threshold of statistical significance was set to 0.05 .

\section{Results}

\section{Linear regression and correlation}

Simple linear regression and correlation between the saccade amplitude and initial head movement were used to evaluate the participant's accuracy in judging the magnitude of the vestibular stimulation (i.e. head movement). This was calculated for all participants, for each fixation point (central $/ 15^{\circ}$ to the right) and for each rotation direction (left / right) (Fig1.). For each condition, a straight line fitted to all the data. The slopes of the relations were 0.80 and 0.53 for the central fixation point, and 0.36 and 0.71 for the $15^{\circ}$ fixation point (left and right rotation respectively). In each condition, the correlations were both positive and significant $(\mathrm{p}<0.001): \mathrm{r}=0.53(\mathrm{n}=109)$ and $r=0.51(n=118)$ for the central fixation point, and $r=0.38(n=106)$ and $r=0.51 \quad(n=119)$ for the $15^{\circ}$ 
fixation point (left and right rotation respectively).

Insert Figure 1 approximately here

We furthermore calculated the individual linear regression parameters between the saccade amplitude and rotation amplitude (see Table 1). The regression slopes for each condition were submitted to a 2 Fixation point position (central $/ 15^{\circ}$ to the right) $\mathrm{x} 2$ Rotation directions (left $/$ right) ANOVA, with repeated measurements on both factors to evaluate the effect of eye position on head rotation perception . The ANOVA revealed a significant main effect of Rotation direction, $\underline{\mathrm{F}}$ $(1,9)=6.83$ as well as a significant Fixation point position $x$ Rotation direction interaction, $\underline{F}(1,9)$ $=5.31$. It revealed a significantly lower slope when the rotation occurred in the opposite direction to the fixation point (i.e. to the left) when the latter was not centered, whereas there was no difference based on rotation direction when the fixation point was centered.

\section{Rotation perception error}

In order to quantify the accuracy of the perceived chair (head) rotation and its reproduction, we calculated the difference (in degrees) between the saccade amplitude and the chair rotation amplitude, hereafter referred to as rotation perception error. A positive value therefore represents an overestimation of the head rotation and a negative value represents an underestimation.

Differences in the oculo-motor task between left and right rotations when the fixation point was not centered $\left(15^{\circ}\right.$ to the right) could affect perception error. Indeed, for the left rotation, participants were forced to produce a further ex-centration of their gaze, while for the right rotation; they made a re-centering saccade (i.e. toward their midline). Furthermore, in this last condition, saccades bigger than $15^{\circ}$ crossed the midline while the others did not. In order to take into account possible effects 
of oculo-motor task on perception error, we identified three types of rotation: (1) around 15 degrees (from 13 to 17 degrees), (2) under 13 degrees and (3) over 17 degrees, then submitted the rotation perception error to a 2 Rotation direction x 3 Type of rotation ANOVA, with repeated measurements on both factors. The ANOVA revealed a main effect of Type of rotation, $\underline{F}(2,18)=27.12$. Its breakdown showed an overestimation for the smallest rotations $\left(+2.76^{\circ}\right.$ mean error for rotations inferior to 13 degrees) and an underestimation for the largest rotations $\left(-4.62^{\circ}\right.$ mean error for rotations superior to 17 degrees), while errors were minimal $\left(-0,54^{\circ}\right.$ mean error) for rotations between 13 and 17 degrees. The ANOVA also revealed a significant Rotation direction x Type of rotation interaction, $\underline{\mathrm{F}}(2,18)=3.86$ illustrated in Figure 2. Its breakdown revealed a significant difference between leftward and rightward rotations, though only for rotations of 13 to 17 degrees. In these cases, leftward rotations were overestimated whereas rightward rotations were underestimated (mean errors of $+1.10^{\circ}$ and $-2.19^{\circ}$ respectively).

Insert Figure 2 approximately here

\section{Discussion}

The main goal of this study was to determine the effect of eye position on the vestibularly derived perception of passive body rotations. Our results indicate that, in line with previous studies (Bloomberg et al. 1988; Bloomberg et al. 1991; Israel et al. 1993; Mergner et al. 1998), participants can perform appropriate ocular saccade in the dark after a step change of head position. Since no visual and auditory information was available, and the head remained fixed on the body, these saccades therefore represent the vestibularly derived perception of the body displacement. We also noted that saccades were less representative of body displacement when the eyes were deviated in the opposite direction to the rotation, suggesting a less accurate and decreased perception of the 
rotation in this condition.

\section{Body rotation perception}

The experimental procedure used in this study was based on the vestibular-memory contingent saccade paradigm in which participants must reproduce the perceived body rotation through an ocular saccade. Our results show a significant positive correlation between the imposed body rotation and the saccade amplitude, indicating an accurate perception of the rotation through vestibular inputs, which is in line with previous findings (Bloomberg et al. 1988; Bloomberg et al. 1991; Israel et al. 1993; Blouin et al. 1995a; Blouin et al. 1997). The slope of the relationship between rotation amplitude and saccade amplitude is however lower in our study (0.67) than in the first studies using this experimental paradigm ( 0.84 and 0.82 respectively in (Bloomberg et al. 1988; Bloomberg et al. 1991)). Threecombined methodological factors can explain these variations: (1) absence of any type of visual feedback, (2) absence of repetition of the stimulation, and (3) presence of a constant velocity plateau during the largest stimulations. (1) In Bloomberg's studies, participants could use extrinsic visual information concerning their position in the room between each rotation since the lights were turned on between the trials. On the contrary, in our study, participants evaluated their displacement through intrinsic vestibular input only as they remained in the dark during the whole experiment (i.e. the rotation, the subsequent saccade and the time before the next trial). This could partially explain our lower slopes. In fact, Blouin et al. (1997) and Israel et al. (1993) obtained lower regression slopes $(0,79)$ using the same type of stimulation as Bloomberg's (5 repetitions of 6 amplitudes), but without any type of visual feedback available. Thus, in Bloomberg's studies, the visual information seems to be used in subsequent trials either as a type of Knowledge of Results or as a way to calibrate the vestibular system. Accordingly, it has been shown that in the presence of visual feedback, error performed during a vestibular-memory contingent saccade paradigm decreased in less than 4 trials (Israel et al. 1999). (2) Also, contrarily to all the previously cited studies (Bloomberg et al. 1988; Bloomberg et al. 1991; Israel et al. 1993; 
Blouin et al. 1997) that used step changes of no more than six angular amplitudes randomly presented several times, we used rotations randomly ranging from 3 to 30 degrees meaning that participants were never submitted twice to the same displacement amplitude. Thus no estimate could be deduced from the past trials that could explain our lower regression slopes compared to Blouin et al. (1997) and Israel et al. (1993). (3) Finally, in the stimulation we used, velocity could remain constant for up to $2 \mathrm{~s}$ during the largest rotation $\left(30^{\circ}\right)$. Thus, owing to the fact that the central time-constant of the peripheral system in human has been estimated at 17.5s (Honrubia et al. 1982), the central vestibular activity could have been decreased by a maximum of $12 \%$ at the end of the $2 \mathrm{~s}$ velocity plateau. That could partly account for the observed underestimation of the perceived rotation and its variability.

To conclude, our results show that, in absence of any type of extrinsic information, one can appropriately evaluate completely random body displacement through mainly vestibular inputs. Furthermore, it seems that any additional extrinsic information about the displacement, even indirect such as visual information of spatial position as it was the case in Bloomberg's studies, can enhance the precision of the whole body rotation perception.

Even though vestibular signal is likely to play a major role in the perception of the rotation, one could argue that the estimation of rotation duration might also have been used to evaluate body displacement. The reason being that in our study as well as in others (Bloomberg et al. 1988; Bloomberg et al. 1991), rotation amplitude was controlled through rotation duration. However, in addition to the fact that a recent study showed that rotation duration is poorly reproduced after passive self-rotation (Israel et al. 2006), the profile of stimulation we used did not allow participants to rely on duration estimation to accurately evaluate body displacement. This is due to the fact that amplitude was not proportional to time (amplitude $=2.5 \times \mathrm{t}^{2}$ for rotations smaller than $10^{\circ}$ and amplitude $=10 \mathrm{x}(\mathrm{t}-1)$ for rotations greater than $\left.10^{\circ}\right)$, nor to velocity. Therefore, it would have been very difficult for participants to estimate the amplitude of body rotation from duration or 
velocity. Whereas in studies using a bell-shaped velocity profile of constant duration (Israel et al. 1993; Blouin et al. 1997), there is a linear relationship between the maximum velocity and the amplitude of rotation which can be used by the participant to estimate body displacement.

Thus, the significant correlation we obtained between imposed body rotation and saccade amplitude can't be explained by duration reproduction, but can only result from rotation perception based on vestibular input. Taken together, these results indicate that one can correctly evaluate body displacement in total darkness through intrinsic inputs only (mainly vestibular).

\section{Effect of eye position on body rotation perception}

During a passive body rotation, the eye position within the orbit appears to play a role in rotation perception. Whilst the perception was equivalent when the eyes were straight-ahead or deviated in rotation direction, this perception seemed less accurate and of less magnitude when the eyes were deviated in the direction opposite to the rotation. In fact, in this latter condition, we observed both significantly lower regression slopes and lower correlation coefficient in the relationship between imposed body rotation and saccade amplitude (see Figure 1). Nonetheless, the correlation remained positive and significant, suggesting that the rotation of the body is still perceived, but in a less effective way. It is well known that the vestibulo-occular response is affected by the position of the eyes within the orbit with a reduced response when the eyes are orientated in the opposite direction to the rotation as stated by Alexander's law (Robinson et al. 1984; Evanoff and Lackner 1986). However, the consequences of this phenomenon on perception remained unexplored. Our results show that perception is affected in a similar way to the neurophysiological signal, which leads us to consider this phenomenon as an extension of Alexander's law to the perceptive side. This would stipulate that the perception of head displacement is reduced when the eyes are deviated in the opposite direction to the rotation. This finding is coherent with observations reported by Evanoff and Lackner (Evanoff and Lackner 1986) where participants experienced difficulty in voluntarily maintaining ocular deviation in the opposite direction to body rotation. Furthermore, the fact that 
eye position within the orbit affects the vestibulo-ocular reflex as well as the perception of body rotation, allows us to postulate on neurological substrate that are responsible for this effect. One can hypothesize that the signal carrying information on eye position, originating from eye proprioception or from corollary discharge, alters the peripheral vestibular signal integration before oculomotor and cortical pathway dissociation.

There is however, an alternative and/or complementary hypothesis which could account for our results. It concerns differences in the oculo-motor task when the eyes are deviated either in the opposite direction to body rotation or in the same direction. In the former condition, a further excentration of the gaze had to be produced, while in the latter condition, it was a re-centration. These re-centration saccades could be expected to be more precise, especially when they ended along a participant's midline, which has been proposed to act as an anchor of the egocentric frame of reference (Jeannerod 1988; Jeannerod and Biguer 1989; Chokron and Bartolomeo 2000; Lhuisset and Proteau 2004). We tested this hypothesis by comparing saccades for different body rotation amplitudes in both rotation directions to evaluate the effect of a person's midline attraction on the saccade production. More precisely, we distinguished between saccades inferior and superior to $15^{\circ}$. When the eyes were deviated in the direction of the rotation, saccades inferior to $15^{\circ}$ remained in the right hemispace while saccades superior to $15^{\circ}$ had to cross someone's midline. If someone's midline plays as an attractor on the saccade production, the former saccades should be hypermetric, the latter ones hypometric and the $15^{\circ}$ saccades, ending along someone's midline, the most precise. We indeed obtained hypermetric saccades for rotations inferior to $15^{\circ}$ and hypometric saccades for rotations superior to $15^{\circ}$ when the eyes were deviated in the direction of the rotation. This could have suggested that someone's midline plays a role as an egocentric reference in the saccade production. However, a similar pattern of results was also present when the eyes were deviated in the opposite direction to the rotation. Furthermore, saccades following a $15^{\circ}$ rotation were no more accurate for the former eye positioning than for the latter. It seems therefore that regardless of the 
direction of eye deviation, smaller rotations are overestimated when larger rotations are underestimated as it has been previously reported by Bloomberg et al (Bloomberg et al. 1988; Bloomberg et al. 1991). This phenomenon can be related to a general psychophysical outcome known as the range effect (Slack 1953) and inducing an estimate biased toward the middle range of all the stimulations.

\section{Conclusion}

In conclusion, we have demonstrated an effect of eye position within the orbit on body rotation perception based solely on intrinsic inputs (i.e. vestibular and somesthetic information). This effect indicates a poorer and decreased perception when the eyes are deviated in the opposite direction to the rotation. This can be related to a physiological phenomenon known as Alexander's law stipulating less intense nystagmus when the gaze is oriented in the opposite direction to the rotation and one can consider the effect we observed as an extension of Alexander's law to perceptive aspects. 


\section{References}

Bloomberg J, Jones GM, Segal B, McFarlane S, Soul J (1988) Vestibular-contingent voluntary saccades based on cognitive estimates of remembered vestibular information. Adv Otorhinolaryngol 41: 71-75

Bloomberg J, Melvill Jones G, Segal B (1991) Adaptive modification of vestibularly perceived rotation. Exp Brain Res 84: 47-56

Blouin J, Gauthier GM, Vandonkelaar P, Vercher JL (1995a) Encoding the position of a flashed visual target after passive body rotations. Neuroreport 6: 1165-1168

Blouin J, Gauthier GM, Vercher JL (1995b) Failure to update the egocentric representation of the visual space through labyrinthine signal. Brain and Cognition 29: 1-22

Blouin J, Gauthier GM, Vercher JL (1997) Visual object localization through vestibular and neck inputs. 2: Updating off-mid-sagittal-plane target positions. J Vestib Res 7: 137-143

Brown JH (1966) Magnitude estimation of angular velocity during passive rotation. J Exp Psychol 72: $169-172$

Chokron S, Bartolomeo P (2000) Correlation between the position of the egocentric reference and right neglect signs in left-brain-damaged patients. Brain Cogn 43: 99-104

Evanoff JN, Lackner JR (1986) Influence of voluntary ocular deviation on vestibular nystagmus. Acta Otolaryngol 102: 450-456

Greenhouse SW, Geisser S (1959) On the methods in the analysis of profile data. Psychometrika 24: 95-112

Honrubia V, Jenkins HA, Baloh RW, Konrad HR, Yee RD, Ward PH (1982) Comparison of vestibular subjective sensation and nystagmus responses during computerized harmonic acceleration tests. Ann Otol Rhinol Laryngol 91: 493-500

Israel I, Fetter M, Koenig E (1993) Vestibular perception of passive whole-body rotation about horizontal and vertical axes in humans: goal-directed vestibulo-ocular reflex and vestibular memory-contingent saccades. Exp Brain Res 96: 335-346

Israel I, Siegler I, Rivaud-Pechoux S, Gaymard B, Leboucher P, Ehrette M, Berthoz A, PierrotDeseilligny C, Flash T (2006) Reproduction of self-rotation duration. Neurosci Lett 402: 244-248

Israel I, Ventre-Dominey J, Denise P (1999) Vestibular information contributes to update retinotopic maps. Neuroreport 10: 3479-3483

Jeannerod M (1988) The neural and behavioral organization of goal-directed movements. Oxford University Press, Oxford

Jeannerod M, Biguer B (1989) [Egocentric reference and represented space]. Rev Neurol (Paris) 145: 635-639 
Jurgens R, Boss T, Becker W (1999) Estimation of self-turning in the dark: comparison between active and passive rotation. Exp Brain Res 128: 491-504

Lhuisset L, Proteau L (2004) Planning and control of straight-ahead and angled planar movements in adults and young children. Can J Exp Psychol 58: 245-258

Mergner T, Nasios G, Maurer C, Becker W (2001) Visual object localisation in space. Interaction of retinal, eye position, vestibular and neck proprioceptive information. Exp Brain Res 141: $33-51$

Mergner T, Schweigart G, Botti F, Lehmann A (1998) Eye movements evoked by proprioceptive stimulation along the body axis in humans. Exp Brain Res 120: 450-460

Metcalfe T, Gresty M (1992) Self-controlled reorienting movements in response to rotational displacements in normal subjects and patients with labyrinthine disease. Ann N Y Acad Sci 656: 695-698

Robinson DA, Zee DS, Hain TC, Holmes A, Rosenberg LF (1984) Alexander's law: its behavior and origin in the human vestibulo-ocular reflex. Ann Neurol 16: 714-722

Siegler I, Viaud-Delmon I, Israel I, Berthoz A (2000) Self-motion perception during a sequence of whole-body rotations in darkeness. Exp Brain Res 134: 66-73

Slack CW (1953) Some characteristics of the 'Range effect'. J Exp Psychol 46: 76-80

Winer BJ (1971) Statistical principles in experimental design. Mc Graw-Hill, New York 


\section{Figure captions}

Fig. 1 Amplitude of saccadic eye movement (perceived rotation amplitude) versus amplitude of whole body rotation (real rotation amplitude) plotted for all participants. Each point is the result of one trial of one participant. Solid lines give the simple regression line fitted to the data.

a. Full data set when the fixation point is located straight ahead. In the left panel are represented the leftward rotations, while the rightward rotations are illustrated in the right panel

b. Full data set when the fixation point is not centered (located $15^{\circ}$ to the right). In the left panel are represented the leftward rotations (i.e. in the opposite direction to the fixation point), while the rightward rotations are illustrated in the right panel (i.e. in the direction of the fixation point).

It is worth noting the inferior slope and inferior Pearson coefficient with a non centered fixation point occurring only when the rotation is in the opposite direction to the fixation point.

Fig. 2 Mean errors of rotation perception (+ standard error of the mean) as a function of rotation amplitude in the non-centered fixation condition. 
a

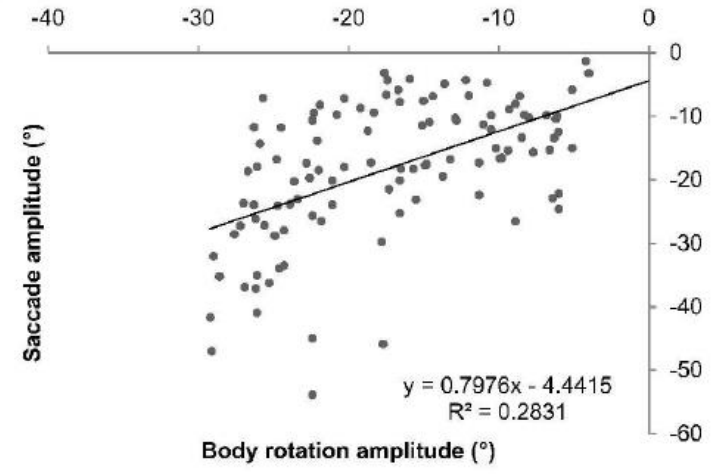

b

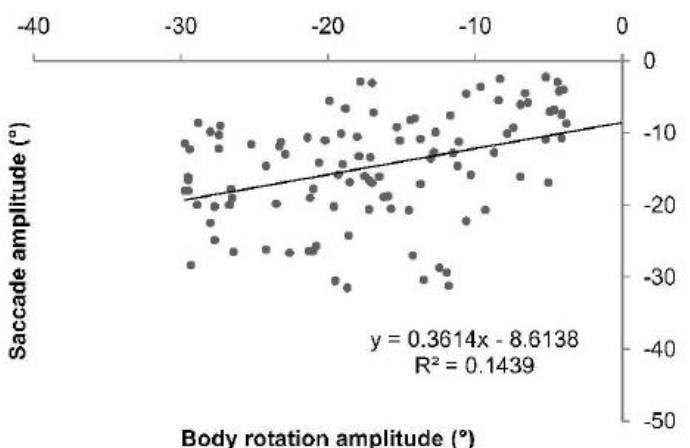

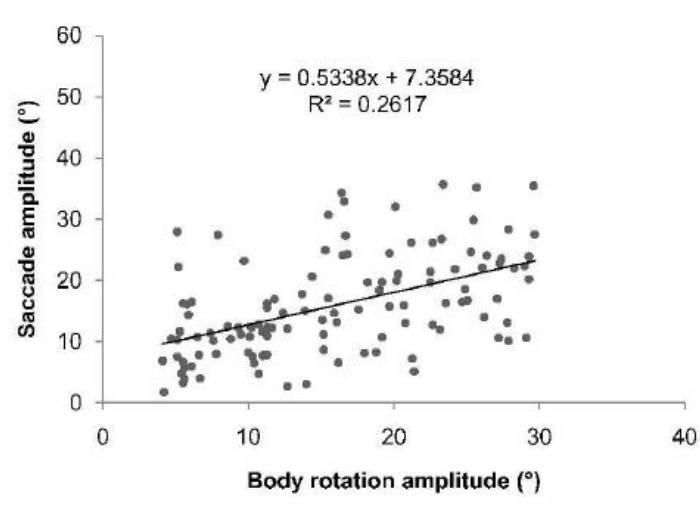

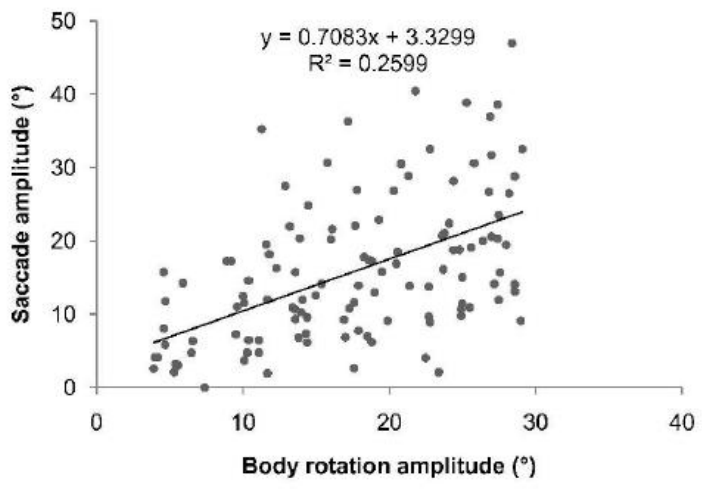

Fig 1 


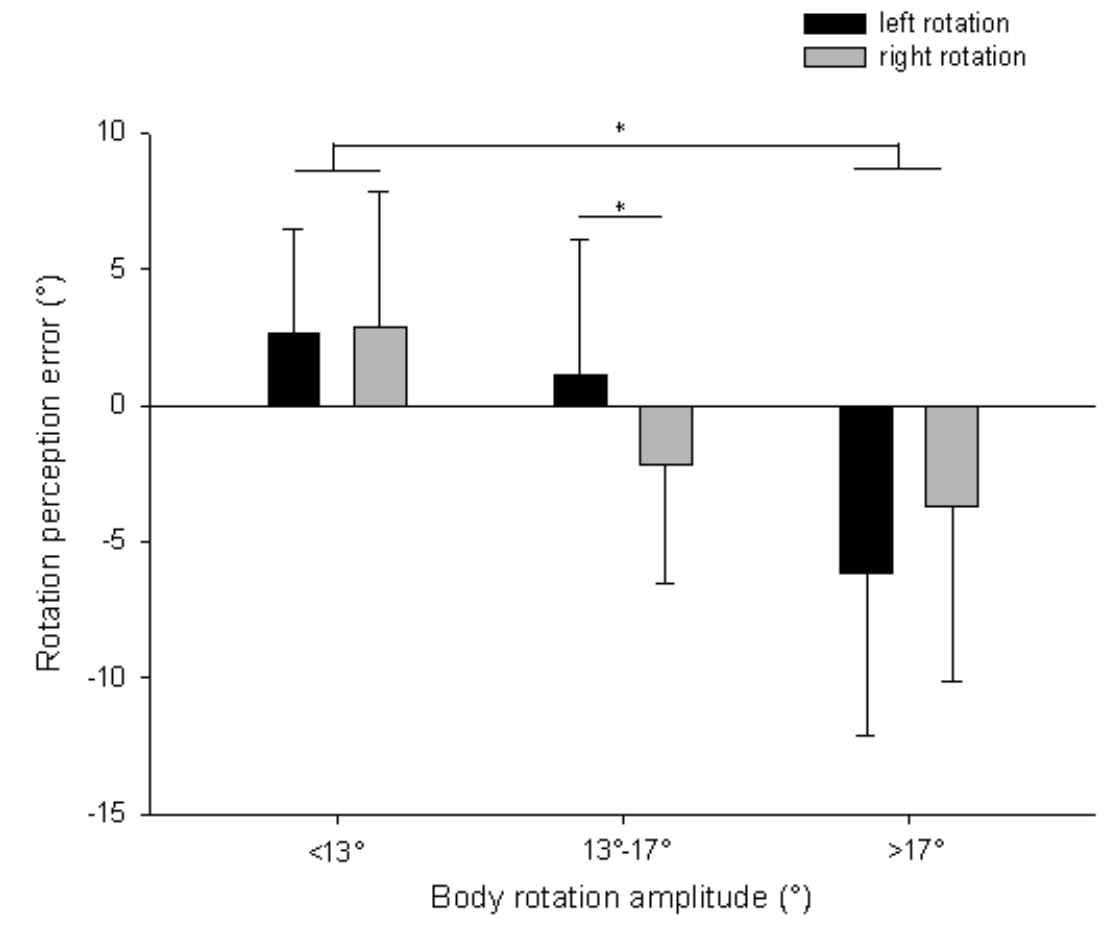

Fig 2 
Table 1. Individual linear regression parameters between the saccade amplitude and the rotation amplitude as a function of fixation point position and rotation direction.

Note the higher individual $r^{2}$ as compared to when all participants are considered in the same linear regression (Fig.1). This is due to interparticipants variability.

\begin{tabular}{|c|c|c|c|c|c|c|c|c|c|c|c|c|}
\hline \multirow[b]{3}{*}{ Participant } & \multicolumn{6}{|c|}{ Centered fixation point } & \multicolumn{6}{|c|}{ Non-centered fixation point $\left(15^{\circ}\right.$ to the right $)$} \\
\hline & \multicolumn{3}{|c|}{ Left rotation } & \multicolumn{3}{|c|}{ Right rotation } & \multicolumn{3}{|c|}{ Left rotation } & \multicolumn{3}{|c|}{ Right rotation } \\
\hline & Slope & Intercept & $\mathrm{r}^{2}$ & Slope & Intercept & $\mathrm{r}^{2}$ & Slope & Intercept & $\mathrm{r}^{2}$ & Slope & Intercept & $\mathrm{r}^{2}$ \\
\hline 1 & 0.56 & 1.73 & 0.78 & 0.51 & 4.23 & 0.30 & 0.52 & -1.89 & 0.78 & 0.74 & -1.21 & 0.88 \\
\hline 2 & 0.64 & -7.11 & 0.44 & 0.69 & 6.01 & 0.73 & 0.15 & -12.44 & 0.57 & 0.35 & 15.11 & 0.15 \\
\hline 3 & 0.34 & 0.06 & 0.64 & 0.34 & 0.25 & 0.75 & 0.19 & -4.69 & 0.18 & 0.29 & -0.03 & 0.32 \\
\hline 4 & 1.20 & -1.38 & 0.67 & 0.74 & 7.23 & 0.63 & 0.55 & -6.76 & 0.49 & 0.99 & -5.67 & 0.42 \\
\hline 5 & 0.52 & -7.74 & 0.60 & 0.76 & 5.13 & 0.72 & 0.34 & -3.34 & 0.58 & 0.45 & 4.92 & 0.74 \\
\hline 6 & 1.02 & -8.38 & 0.81 & 0.66 & 5.15 & 0.58 & 0.24 & -23.07 & 0.15 & 0.65 & 6.40 & 0.39 \\
\hline 7 & 0.17 & -7.54 & 0.21 & 0.46 & 6.47 & 0.40 & 0.17 & -6.31 & 0.15 & 0.16 & 6.07 & 0.36 \\
\hline 8 & 1.18 & -12.72 & 0.57 & 0.88 & 9.54 & 0.37 & 1.07 & -5.18 & 0.68 & 1.21 & 5.66 & 0.46 \\
\hline 9 & 0.60 & -8.40 & 0.53 & 0.53 & 6.10 & 0.53 & 0.62 & -7.08 & 0.86 & 0.81 & 7.29 & 0.55 \\
\hline 10 & 0.32 & -13.56 & 0.56 & 0.51 & 13.35 & 0.22 & 0.41 & -5.16 & 0.42 & 0.81 & 4.15 & 0.45 \\
\hline " mean & $\overline{c 0.65}$ & & & "0.61 & & & 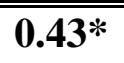 & & & 0.65 & & \\
\hline
\end{tabular}

$* \mathrm{p}>0.05$ 